\title{
GRUPOS INTERACTIVOS EN AULA Y EL INCREMENTO DE LOS RESULTADOS DE APRENDIZAJE EN LENGUAJE EN UN COLEGIO VULNERABLE ${ }^{1}$
}

\author{
Marcela Núñez Solís* \\ Universidad de Concepción (UdeC), Los Ángeles- Chile \\ Doctoranda Universidad de Sevilla (US), Sevilla- España \\ Cristhian Espinoza Navarrete*" \\ Universidad de Concepción (UdeC), Los Ángeles- Chile \\ Carmen Acuña Zúñiga ${ }^{* * *}$ \\ Universidad de Concepción (UdeC), Los Ángeles- Chile \\ Lilian Vargas Villar**** \\ Universidad de Concepción (UdeC), Los Ángeles- Chile \\ Irma Lagos Herrera ${ }^{* \cdots * * *}$ \\ Universidad de Concepción (UdeC), Los Ángeles- Chile
}

RESUMEN: Este artículo presenta los resultados de un estudio realizado entre los años 2013 y 2014 en un colegio vulnerable ${ }^{2}$ en Los Ángeles, Chile. Ese estudio consistió en la incorporación de grupos interactivos en aula en primero básico ${ }^{3}$ en Lenguaje. El objetivo fue determinar el incremento de los resultados de aprendizaje en dicha área. Se utilizó un enfoque cuantitativo, con diseño preexperimental y aplicación de CL-PT ${ }^{4}$ en el nivel señalado, antes y después de la intervención. El trabajo, además, consideró la incorporación en aula de colaboradores de aprendizaje: apoderados, alumnos de cursos superiores del mismo colegio, alumnas de pregrado y docentes de la Universidad. Los grupos interactivos involucran un cambio de paradigma que conlleva un fuerte compromiso social. Los resultados indican que se produjo un incremento significativo entre el pretest y postest (CL-PT), lo que implicó una mejora en el desarrollo del lenguaje.

Palabras clave: Grupos Interactivos. Colaboradores de Aprendizaje. Modelo Dialógico. Lenguaje.

"Magíster en Educación. Alumna Programa de Doctorado en Educación, Universidad de Sevilla, España. Docente de la Universidad de Concepción. E-mail: < marcenun@udec.cl> .

"Doctor en Literatura Latinoamericana. Docente de la Universidad de Concepción.

E-mail: < cristhianbernes@udec.cl>.

"*"Doctora en Ciencias Sociales. Docente de la Universidad de Concepción.

E-mail: < carmenclaudiacu@udec.cl>.

"***Magíster en Didáctica de la Matemática. Docente de la Universidad de Concepción.

E-mail: < lilivargas@udec.cl>.

".*.*Magíster en Educación con mención en currículum. Docente de la Universidad de Concepción.

E-mail: <ilagos@udec.cl>. 


\section{GRUPOS INTERATIVOS EM SALA DE AULA E O PROGRESSO DOS RESULTADOS DE APRENDIZAGEM EM LINGUAGEM EM UMA ESCOLA EM SITUAC̣̃̃O DE VULNERABILIDADE}

RESUMO: Este artigo apresenta os resultados de um estudo realizado entre 2013 e 2014 em um colégio em situação de vulnerabilidade ${ }^{5}$ em Los Ángeles, Chile. Esse estudo consistiu na incorporação de grupos interativos em sala de aula no primeiro ano básico ${ }^{6}$ em Linguagem. O objetivo foi delinear o progresso dos resultados de aprendizagem nessa área. Utilizou-se um enfoque quantitativo, com uma configuração pré-experimental e aplicação de CL-PT ${ }^{7}$ no nível escolar já mencionado, antes e depois da intervenção. O trabalho, além disso, considerou a incorporação em sala de aula de colaboradores de aprendizagem: pais ou responsáveis pelos alunos; alunos de anos posteriores do mesmo colégio; alunas de graduação; e docentes da Universidade. Os grupos interativos envolvem uma mudança de paradigma que implica um forte compromisso social. Os resultados indicam que se produziu um progresso significativo entre o pré-teste e o pós-teste (CLPT), o que acarretou uma melhora no desenvolvimento da linguagem.

Palavras-chave: Gruposw Interativos. Colaboradores de Aprendizagem. Modelo Dialógico. Linguagem.

\section{INTERACTIVE GROUPS IN THE CLASSROOM AND THE LANGUAGE LEARNING PROGRESS IN A SCHOOL IN VULNERABILITY SITUATION}

ABSTRACT: This article presents the results of a study performed during the 2013 to 2014 period in a "vulnerable" school in Los Angeles, Chile. The study has consisted of the incorporation of interactive groups in first grade language classes. The aim was to determine increased learning outcomes in this area. A quantitative approach was used with a pre-experimental design and the application of reading comprehension and text production tests in the mentioned grade, before and after the intervention. Furthermore, this study also considered the incorporation of learning collaborators into the classroom: parents or guardians, students in higher grades in the same school, undergraduate students, and university professors. The interactive groups involve a paradigm change that leads to a strong social commitment. The results indicate that a significant increase was produced between the pre and post test, which led to an improvement in language learning. Keywords: Interactive Groups. Learning Collaborators. Dialogic Model. Language. 


\section{INTRODUCCIÓN}

Paulo Freire (1970) denominaba educación bancaria a una forma de concebir la educación como una relación vertical en la cual el educador otorga los conocimientos y los educandos los reciben pasivamente. En este tipo de modelo pedagógico existe una división de los roles de educador y educando.

En este mismo sentido, en Latinoamérica, nuestras nacientes sociedades postmodernas han privilegiado un desarrollo reduccionista de los seres humanos, implementándose estrategias de enseñanza en las cuales permanece el modelo "bancario" señalado por Freire y formas "objetivas" de evaluación. En ese contexto, lo que importa es avanzar en los contenidos. La consideración holística y global del ser humano se pierde en objetivos focalizados, contenidos especializados y parcializados sobre los que trabajan los aprendices desde el nivel preescolar, con fuerte demanda de energía que genera cansancio, aburrimiento, desmotivación por aprender, contrapuesto al dinamismo de la vida de los niños. Es así como "las críticas a la educación tradicional se han centrado fundamentalmente en la escasa o nula presencia del diálogo en el aula, entendido éste como intercambio comunicativo equitativo y respetuoso en una interacción equilibrada." (VELASCO; ALONSO, 2008, p. 462).

Teniendo en cuenta esta realidad y a la luz de las investigaciones en el ámbito de las prácticas educativas y de las ciencias del lenguaje, desde los años 90, el Ministerio de Educación de Chile (MINEDUC) ha venido realizando una serie de propuestas metodológicas que pretenden introducir prácticas dialógicas y colaborativas en la enseñanza del lenguaje desde la educación preescolar, a partir de una perspectiva constructivista (INOSTROZA, 1996). Sin embargo, dichas prácticas, salvo excepciones, no han logrado permear la actividad de los docentes que trabajan en el sistema educacional chileno, quienes continúan reproduciendo modelos de enseñanza conductista del lenguaje. Esto debido a que, entre otras variables, para los profesores, según lo señalado en un estudio que buscaba abordar la reticencia de los docentes al cambio,

[...] el hecho de abandonar sus formas tradicionales de enseñanza les resulta molesto y en ocasiones hasta doloroso, porque lo nuevo provoca confusión y surge el temor de arriesgar la credibilidad profesional, por lo tanto, se oponen abiertamente al cambio. Aunque, la mayoría de las veces, se aducen responsabilidades a terceros: características sociales de los estudiantes, recursos de que dispone la unidad educativa, entre otros. (INOSTROZA, 1996, p. 29). 
En este contexto, la enseñanza y el aprendizaje en el área de lenguaje son deficitarios, y así lo demuestran los resultados que entrega la Agencia de Calidad de la Educación a nivel país (2014) para el SIMCE ${ }^{8}$ 2013, que ubican al segundo básico del año 2013 mayoritariamente en nivel de aprendizaje elemental, de los tres niveles que se establecen en esta prueba (adecuado, elemental e insuficiente).

Además, los resultados de las evaluaciones internacionales demuestran una clara distancia en los puntajes obtenidos por los estudiantes chilenos en lenguaje en comparación a los otros países OCDE, 9 que obtienen altas puntuaciones en estas pruebas (OCDE, 2012). Esta asimetría se superaría si se innovaran las estrategias de enseñanza del modelo tradicional de educación y se hicieran esfuerzos por construir sistemas educacionales menos excluyentes y más participativos. Este es justamente el sentido de la innovación educativa desarrollada en esta investigación, que se basa en el trabajo en aula con grupos interactivos e implica, entre otras cosas, actos comunicativos dialógicos basados en el diálogo igualitario (OLIVER; GATT, 2010). La institución educativa involucrada en esta investigación fue un colegio particular subvencionado ${ }^{10}$ de alta vulnerabilidad, específicamente un primer año básico.

Este modelo dialógico de grupos interactivos deviene del trabajo de Comunidades de Aprendizaje, iniciado en el País Vasco en el año 1995 (FERRADA, 2012). Se trata de una alternativa que contribuye a la inclusión y a la participación del estudiantado desde su contexto de vida, en que los mismos docentes elaboran las acciones pedagógicas a seguir en conjunto con los colaboradores de aprendizaje, ${ }^{11}$ lo que propicia mejores instancias de aprendizajes y de desarrollo personal y social, aun en los grupos sociales vulnerables.

Las investigaciones demuestran que, trabajando a partir de este modelo dialógico de grupos interactivos, se acelera el aprendizaje y se fomenta la solidaridad, ya que, si antes era un solo profesor el que trabajaba con 30 o 35 alumnos, ahora es ese mismo profesor quien tutoriza la clase y cinco personas adultas que trabajan con los grupos de manera simultánea (ELBOJ et al. 2009). De esta manera, asumir la pedagogía desde una perspectiva dialógica implica que la educación se convierte en "un medio para promover interacciones humanas dirigidas a transformar las propias construcciones intersubjetivas de quienes participan en el acto educativo al interior de una comunidad con predominio de la racionalidad comunicativa." (FERRADA; FLECHA, 2008 p. 59). Se logra, así, construir nuevos significados de manera conjunta, relacionados con ciertas decisiones educativas, tales como 
organización del espacio y tiempo, asignación de roles, entre otros. Todo lo cual propicia iniciar un proceso de transformación en la escuela.

En este sentido, es importante destacar que, para poder lograr el éxito en el centro educativo donde se trabajó y responder a los objetivos de la investigación propuesta, se consideraron las necesidades de dicho centro y no se impuso un modelo rígido, dándose las posibilidades de que el propio equipo educativo del colegio analizara y decidiera qué era pertinente para su realidad y determinara los cambios que requería realizar de acuerdo a su contexto. Al respecto, Baroccio (2000 citado por GUERRERO, 2005) señala que, en muchos centros educativos, cuando se propone realizar una innovación desde afuera, existe la tendencia a negarse a él o aceptarlo. Ambas serían formas de resistir al cambio propuesto, debido a que una institución que realmente quiere un cambio debe tener la posibilidad de ser protagonista y poder elegir todo aquello que tiene relevancia para la institución educativa.

Siguiendo esta idea, el equipo investigador decidió proponer e incorporar solo algunos de los aspectos de las Comunidades de Aprendizaje, teniendo en cuenta la duración de la intervención y los escasos tiempos que los profesores del colegio tenían para el trabajo fuera de aula. Esto limitaba las posibilidades de convocar a más del $90 \%$ de la comunidad del establecimiento, tal como lo plantean los proyectos de Comunidades de Aprendizaje. Fue así como se reestructuraron y acotaron las fases que proponen estas Comunidades, y se focalizó el trabajo en grupos interactivos de aula y reuniones dialógicas en las que participaban los colaboradores de aprendizaje.

El objetivo general del presente estudio es determinar el posible incremento de los resultados de aprendizaje en el área de lenguaje en primero básico en un colegio con alta vulnerabilidad, en la ciudad de Los Ángeles, luego de la incorporación de un modelo dialógico de grupos interactivos. Para esto se plantearon tres objetivos específicos que se resumen en: determinar el posible incremento de los resultados de aprendizajes en niños de primero básico en las siguientes dimensiones: comprensión lectora, producción de textos y manejo de la lengua.

\section{BREVE REFERENTE TEÓRICO}

En el contexto escolar, las interacciones que se deberían producir son de carácter dialógico, sin embargo, la praxis y los estudios demuestran otra cosa. En este sentido, Echeverría (2004, p. 
79) señala que "una de las características de la escuela tradicional es su organización jerárquica, donde el maestro es el que sabe y decide qué hacer y los alumnos escuchan, aprenden y obedecen".

Por su parte, Burbules (1999, p. 13) señala que "iniciar un diálogo con éxito es algo que debemos aprender a hacer por medio de la práctica, no según una receta o un algoritmo". De la misma forma, este autor platea que el diálogo es una relación en la que entramos, no es algo que empleemos. Si consideramos el diálogo de esta forma, es decir, como una relación, se destacan aspectos que están fuera de nosotros y que, por tanto, nos modifican.

Consecuentemente, en el área de lenguaje, desde los años 90 en Chile y otros países de Latinoamérica, se han intentado introducir prácticas colaborativas y con enfoques "holísticos" en la enseñanza de la lectura y la escritura. Dichas propuestas han buscado renovar y superar la mirada, marcadamente conductista, presente en la enseñanza de estas habilidades tan cruciales en el desarrollo de los seres humanos contemporáneos (JOLIBERT, 2003; JOLIBERT; SRAÏKI, 2009; FONS, 2004; DÍEZ, 2004). Estas propuestas incorporan la complejidad de la actividad lectora situándola en la intersección de varios ejes: conocimiento del léxico y los procesos de lectura; conocimiento de la lengua escrita; teorías del aprendizaje; interacciones adultos-niños y las relaciones de poder que se establecen entre ellos; y el funcionamiento de la institución escolar en torno al encuentro del niño con la lectura (JOLIBERT, 2003).

Asimismo, la escritura es entendida como una actividad de alta complejidad en la que interviene una gran cantidad de microhabilidades que se despliegan en el acto de escribir (CASSANY, 2008). En este sentido, Tolchinsky (1993 citado por DÍEZ, 2004, p. 23) señala,

\footnotetext{
La escritura posee una doble naturaleza: como sistema de notación y como medio de comunicación. Como sistema de notación hace referencia a un conocimiento de la escritura en sí, de sus propiedades formales. En cuanto medio de comunicación se refiere a sus propiedades instrumentales, que sirve para escribir recetas, noticias, cartas, etc.
}

Desde esta perspectiva, la adquisición del lenguaje escrito se logra en la reflexión sobre y en contacto con diversos materiales y tipos de textos, los que muestran la variedad y las propiedades del lenguaje escrito. Así, el estudiante en contacto con estos materiales diversos "construirá no solo sus ideas personales acerca del sistema de escritura sino también sobre la estructura del lenguaje escrito, sus diferencias con el lenguaje oral, las características de los diferentes tipos de textos, etc." (DÍEZ, 2004, p. 24). 
Por su parte, Parodi (2005) considera relevante, en términos de la enseñanza, la integración de las actividades de lectura y escritura. Esto basado en las investigaciones psicolingüísticas de Eisterhold (1991) y Shanahan (1984) (citados por PARODI, 2005), quienes proponen un modelo "bidireccional" de integración de lectura y escritura. Este modelo postula que las relaciones entre estos procesos se encuentran en una constelación interrelacionada que utiliza un sustrato de conocimiento común, que, sin embargo, no "recarga" de forma significativa "el sistema cognitivo del sujeto" (PARODI, 2005, p. 73). Esto está en concordancia con lo señalado por las investigaciones de Van Dijk y Kintsch (1983 citado por PARODI, 2005, p. 73), quienes puntualizan que "la conexión entre lectura-escritura ahorraría energía del sistema cognitivo del lector/escritor, pues la posibilidad de estrategias comunes de base permitiría una mejor distribución de los recursos energéticos en el procesamiento discursivo".

La señalada incorporación de prácticas renovadas en la enseñanza del lenguaje en Chile se objetiva en la incorporación del denominado "Enfoque equilibrado o integrado", que buscaba, desde el "Programa de las 900 Escuelas" en 1990 hasta los programas de Educación General Básica, ${ }^{12}$ integrar al "modelo de destrezas", que asumía el desarrollo de la lectura como la suma de destrezas que debían aprenderse de forma separada y progresiva, un "modelo holístico", basado en la inmersión temprana en la lectura (ALLIENDE; CONDEMARÍN, 2002). Entre las características que se destacan de este enfoque están: currículum centrado en el niño, enfocado en los intereses de los estudiantes; enfoque de la enseñanza, centrada ésta en la búsqueda de significados de la lengua a través de experiencias con textos relacionados; materiales diversos, significativos y a elección; ambiente de la sala de clases letrados, donde la lectura y escritura son herramientas para resolver problemas; contextos colaborativos entre pares, donde las "interacciones colaborativas entre los estudiantes son un elemento crucial para el desarrollo y enseñanza del lenguaje." (ALLIENDE; CONDEMARÍN, 2002, p. 112). Este "enfoque equilibrado o integrado" fue el utilizado en la incorporación de grupos interactivos de la presente investigación, pues el equipo involucrado del colegio así lo solicitó, para mantener las exigencias del Ministerio de Educación de Chile (MINEDUC).

Así, en el trabajo de campo, se buscó integrar las actividades de lectura y escritura por medio de la lectura dialógica de los textos, que pasan a ser tratados por personas de la misma cultura y de otras (profesores, madres, alumnas de pregrado de la Universidad, alumnos 
de cursos superiores de la misma escuela). De esta manera, se evita la lectura monocultural, lo que contribuye a que la lectura adquiera sentido para los niños y, por lo tanto, puedan motivarse (AGUILAR et al., 2010).

\subsection{Comunidades de Aprendizaje}

Según Flecha (1997), las Comunidades de Aprendizaje apuestan por ser un cambio en la sociedad, llevándola a ser más justa e igualitaria. El punto central de este cambio se genera con el establecimiento del diálogo entre pares y entre los diversos agentes, sin importar su "estatus" dentro de la comunidad educativa. Una comunidad de aprendizaje se define como:

[...] un proyecto de transformación social y cultural de un centro educativo y de su entorno para conseguir una sociedad de la información para todas las personas basada en el aprendizaje dialógico mediante la educación participativa de la comunidad que se concreta en todos sus espacios, incluida el aula. (Valls, 2000, p. 8).

Este proyecto de transformación social y educativa fundamenta sus acciones en la investigación. Es por esto que el Centro Especial de Investigación en Teorías y Prácticas Superadoras de las Desigualdades (CREA) de la Universidad de Barcelona, como resultado de investigaciones sobre las mejores teorías y prácticas a nivel internacional para la superación del fracaso escolar y el aumento de la cohesión social, lleva a cabo el proyecto de reforma de centros educativos en Comunidades de Aprendizaje (FERRADA; FLECHA, 2008).

Tras su éxito en 1995 en el País Vasco, el proyecto se extendió a otras escuelas de esta región española recibiendo apoyo del gobierno local. Posteriormente otras instituciones iniciaron el proceso de transformación en Comunidades de Aprendizaje y en 2008 ya había un total de 52 centros educativos en cuatro comunidades autónomas que funcionaban en España. Luego, esta nueva forma de hacer pedagogía se extendió a cuatro Comunidades de Aprendizaje en Brasil y dos en Chile.

Los principios del aprendizaje dialógico que rigen las Comunidades de Aprendizaje (FLECHA, 2008; AUBERT et al., 2010; ELBOJ et al., 2009) son los que se mencionan a continuación:

a) Diálogo igualitario: con el fin de posibilitar la construcción de significados, se utilizan argumentos y pretensiones de validez. De esta forma, se facilita la ayuda eficaz y profunda en el aprendizaje de nuevas destrezas requeridas por la sociedad del conocimiento. 
b) Inteligencia cultural: es posible entender la inteligencia como un potencial cognitivo, por lo tanto, moldeable, aprendible, transformable y que se "desarrolla en función de las oportunidades que se crean en cada contexto social y cultural." (AUBERT et al., 2010, p. 177).

c) Transformación: el sentido de transformación está vinculado al resto de los principios en la medida en que se pretende transformar la realidad en lugar de adaptarse a ella. De esta manera se busca superar, a través del diálogo, las relaciones de poder con el fin de construir un conocimiento conjunto.

d) Dimensión instrumental del aprendizaje: el aprendizaje de los conocimientos académicos y el desarrollo de herramientas sociales son elementos que las Comunidades de Aprendizaje ofrecen sin realizar discriminación de ningún tipo.

e) Creación de sentido: las Comunidades de Aprendizaje deben ser capaces de crear un ambiente en el que se promueva la reflexión, la búsqueda y construcción de sentido por parte de los participantes.

f) Solidaridad: es un elemento fundamental y es una práctica que debe estar presente en todo proceso educativo, ya que busca mejorar las condiciones de vida para todas las personas sin distinciones. Por tanto, una educación solidaria tiene "que ofrecer los máximos aprendizajes y de la máxima calidad a todos y cada uno de los y las estudiantes." (AUBERT et al., 2010, p. 223).

g) Igualdad de diferencias: este principio supera a la igualdad homogeneizadora y apuesta por una educación que considere las diferencias culturales y personales. La participación del profesor en el aula requiere de otros agentes colaboradores, debido a que el contexto de estos entrega diversidad de oportunidades de aprender, lo que enriquece los aprendizajes de los niños.

\subsection{Procesos y fases de las Comunidades de Aprendizaje}

Es importante destacar que el trabajo de Comunidades de Aprendizaje debe realizarse considerando dos grandes procesos, uno de transformación y el otro de consolidación, los cuales están compuestos, a su vez, por diversas fases (FLECHA; LARENA, 2008):

a) El proceso de transformación está compuesto por las siguientes fases: 
- Sensibilización: considera la presentación a la comunidad educativa de los conceptos que orientan las Comunidades de Aprendizaje. Para esto se realizan reuniones informativas y sesiones de reflexión.

- Toma de decisión: se trata de la decisión conjunta de la comunidad sobre la puesta en marcha del proyecto.

- Sueño: consiste en la proyección de los aprendizajes y de la escuela que se quiere. Se superan las bajas expectativas sobre los alumnos que suelen encontrarse en estos contextos.

- Análisis contextual y selección de prioridades: considera el conocimiento del contexto en el que se inserta la escuela, sus recursos, el alumnado, el profesorado, el fracaso escolar, entre otros. Posteriormente se analizan, debaten e identifican aquellos datos que se deben transformar.

- Planificación de los aspectos a transformar: contempla actividades relativas a las acciones que se desean realizar para lograr las transformaciones.

b) El proceso de consolidación consiste en la puesta en marcha del trabajo que se realiza con la formación de familiares y grupos interactivos. Este proceso implica dos fases:

- Formación de familiares: tiene como objetivo aumentar el aprendizaje instrumental para participar de mejor forma en las Comunidades de Aprendizaje. Se busca en este sentido "el reconocimiento e inclusión del saber popular, la creación de nuevos conocimientos, el cuestionamiento de tradiciones y creencias y la transformación personal y social." (FLECHA; LARENA, 2008, p. 60).

- Formación de grupos interactivos: se trata de una reorganización del aula en grupos, y cada uno de estos (cuatro o cinco grupos) queda a cargo de un colaborador de aprendizaje. En el aula, cada grupo trabaja de forma dialógica y colaborativa, promoviendo un aprendizaje individual y colectivo, en el que se pretende desarrollar la responsabilidad, la motivación y las altas expectativas.

Es necesario destacar que, de acuerdo a experiencias encontradas en la literatura revisada, se puede señalar que la creación de grupos interactivos homogéneos no ofrece resultados positivos. Por el contrario, en la sala de clases deben conformarse los grupos de 
manera heterogénea considerando la diversidad, lo cual favorece la mejora en los resultados académicos y promueve el éxito en todos los niños. Tanto los profesores como los alumnos que han participado de los grupos interactivos señalan que una de las principales ventajas de esta organización es que se intensifican las actividades y, por lo tanto, se acelera el desarrollo de aprendizaje en los niños. De esta manera los alumnos logran desarrollar experiencias educativas que no son capaces de realizar en salas de clases con organización tradicional (VALLS; KYRIAKIDES, 2013).

Los paradigmas tradicionales de la educación sitúan al profesor y al alumno en posiciones de poder verticales. En el caso de los grupos interactivos, estos involucran una distribución distinta de los roles, pues el poder se distribuye de forma horizontal y el docente pasa de ser un agente único del proceso de enseñanza y aprendizaje a tener un rol de coordinador de los colaboradores de aprendizaje. Además, pasa de ser el único que determina las planificaciones a ser un negociador de las demandas pedagógicas de sus alumnos, apoderados, comunidad en general, que serán consideradas en el trabajo de aula y fuera de ella. Por su parte, el alumno deja de ser un receptor de conocimientos y normas establecidas y se transforma en un protagonista de la construcción dialógica del conocimiento (FERRADA; FLECHA, 2008).

En Chile, Ferrada (2012) propone un proyecto de intervención pedagógica denominado Enlazando Mundos. Este toma el trabajo curricular del aprendizaje, didáctico y evaluativo a partir de la construcción intersubjetiva del conocimiento, que implica la construcción colectiva mediante acuerdos dialógicos, entre los diferentes agentes implicados en el quehacer pedagógico. Este proyecto se lleva a cabo en escuelas públicas de la región del Biobío, desde el año 2005. Se caracteriza por estar a cargo de los propios agentes participantes, quienes se han puesto de acuerdo en dar inicio a procesos de profunda transformación pedagógica:

[...] mediante el establecimiento de redes de colaboración entre la Universidad, escuela y la comunidad a fin de conseguir que todos los niños y niñas provenientes de sectores vulnerables tengan las mismas oportunidades y resultados de aprendizaje que los niños que provienen de familias académicas. (FERRADA, 2012, p.19).

Todo lo anterior es coherente con lo planteado por Freire (1970) en torno a que el diálogo reivindica la opción democrática de los docentes y promueve un aprendizaje libre y crítico. Se niega, desde esta mirada, el autoritarismo y se afirma tanto la autoridad como la libertad. 


\section{METODOLOGÍA DE LA INVESTIGACIÓN}

El presente estudio tiene un enfoque cuantitativo, con aplicación de un instrumento de medición de lenguaje antes (abril, 2014) y después (noviembre, 2014) de la conformación de grupos interactivos en la asignatura de Lenguaje en primer año básico. Lo anterior, con el propósito de determinar el incremento en los resultados de aprendizaje de comprensión lectora, producción de textos y manejo de la lengua, por medio de la prueba CL-PT (MEDINA; GAJARDO, 2012).

Es una investigación con diseño preexperimental, dado que se trabajó con grupos intactos ya conformados. Además, por tratarse de un colegio particular subvencionado con características únicas, en la ciudad de Los Ángeles, en cuanto a: alto índice de vulnerabilidad social y bajo puntaje SIMCE en lenguaje.

\subsection{Población y Muestra}

La población está constituida por colegios de la zona urbana de la cuidad de Los Ángeles, Chile, con alta vulnerabilidad social y bajo puntaje en la prueba SIMCE.

La muestra seleccionada es no probabilística, intencionada, considerando los siguientes criterios de inclusión:

a) bajos puntajes en la prueba SIMCE de Lenguaje;

b) centro educativo dispuesto a involucrarse activamente en este proyecto de innovación en la asignatura de Lenguaje.

La muestra está constituida por alumnos de primer año básico de un colegio particular subvencionado con un porcentaje de 81,01 y 100\% de los estudiantes en condición de vulnerabilidad social. El nivel seleccionado consta de 17 alumnos, entre ellos 11 hombres y 6 mujeres, de los cuales el 15\% corresponde a alumnos repitentes y un $45 \%$ de alumnos con NEE. ${ }^{13}$

Este colegio se encuentra situado al sur de la ciudad de Los Ángeles, en un sector con alto riesgo social, y atiende a una población de bajo recurso económico y bajo nivel de escolaridad. En este contexto los padres no cancelan matrícula ni mensualidad.

En cuanto al nivel y la proyección del colegio, este es, como ya se indicó, el establecimiento particular subvencionado con más bajo puntaje SIMCE obtenido dentro de la comuna de Los Ángeles, en el año 2013. El Gráfico 1 muestra los porcentajes de logros obtenidos por el colegio en compresión lectora. 
GRÁFICO 1 - Porcentaje de estudiantes del establecimiento en cada nivel de aprendizaje, según SIMCE $2^{\circ}$ básico -2013

Figura III. Porcentaje de estudiantes del establecimiento en cada Nivel de Aprendizaje, según Simce 2. ${ }^{\circ}$ básico 2013
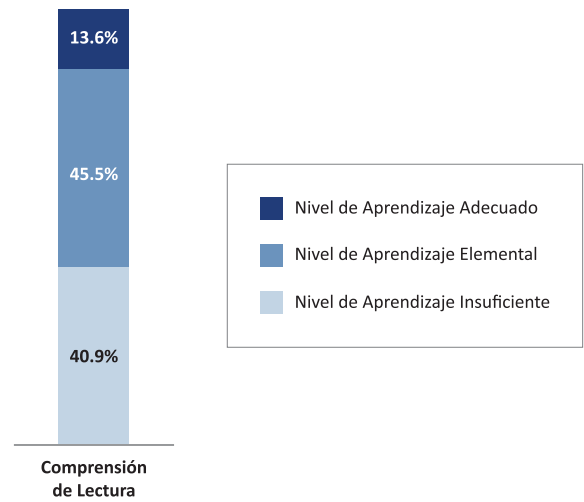

Figura III. Gráfico que muestra la distribución de estudiantes del establecimiento en cada Nivel de Aprendizaje para lectura, según el resultado de la prueba Simce Comprensión de Lectura 2. ${ }^{\circ}$ básico 2013.

Nota: Dado que los porcentajes están aproximados, pueden no sumar exactamente $100 \%$.

Fuente: AGENCIA DE CALIDAD DE LA EDUCACIÓN, 2014.

\subsection{Instrumentos de recolección de datos}

Las pruebas CL-PT (MEDINA; GAJARDO, 2012) son un instrumento actualizado y estandarizado que permitió evaluar a los niños de la muestra, con el fin de determinar el posible incremento en los resultados de aprendizaje de comprensión lectora, producción de textos y manejo de la lengua. Dicha prueba aborda diversas habilidades que están declaradas en el modelo "interactivo y sociocultural de aprendizaje del lenguaje” (MEDINA; GAJARDO, 2012, p.83), así como en los Programas de Estudio y Mapas de Progreso establecidos por el Ministerio de Educación de Chile.

Respecto a la dimensión manejo de la lengua, es fundamental señalar que está relacionada con rasgos que apuntan a determinar conocimientos tanto de comprensión lectora como de producción de textos y que pueden predecir el comportamiento en dichas dimensiones. El Cuadro 1 muestra que, para cada una de estas dimensiones, se definen cinco niveles de desempeño, con su correspondiente porcentaje de logro y descripción (MEDINA; GAJARDO, 2012). 
CUADRO 1 - Niveles de desempeño

\begin{tabular}{|l|l|l|}
\hline Porcentaje de logro & Nivel de Desempeño & \multicolumn{1}{|c|}{ Descripción } \\
\hline $81-100$ & Muy Desarrollado & $\begin{array}{l}\text { Amplio predominio de las fortalezas } \\
\text { sobre las necesidades de aprendizaje }\end{array}$ \\
\hline $61-80$ & Desarrollo Satisfactorio & $\begin{array}{l}\text { Predominio de fortalezas sobre las } \\
\text { necesidades de aprendizaje }\end{array}$ \\
\hline $41-60$ & En Desarrollo & $\begin{array}{l}\text { Necesidades de aprendizaje son } \\
\text { semejantes a fortalezas }\end{array}$ \\
\hline $21-40$ & Emergente & $\begin{array}{l}\text { Aún predominan necesidades de } \\
\text { aprendizaje por sobre fortalezas }\end{array}$ \\
\hline 0.20 & No Desarrollado & $\begin{array}{l}\text { Amplio predominio de necesidades de } \\
\text { aprendizajes por sobre fortalezas }\end{array}$ \\
\hline
\end{tabular}

Fuente: MEDINA; GAJARDO, 2012, p.82.

\subsection{Análisis de datos}

Para analizar los datos, se verificaron los cambios obtenidos entre el pretest y postest, respecto a los efectos de la conformación de grupos interactivos en el nivel involucrado. Para esto, primero se redujeron los datos que componen la prueba de Comprensión Lectora y Producción de Textos (CL-PT). Luego se realizaron comparaciones entre el puntaje bruto de pre- y postest en las tres dimensiones que involucra esta prueba: comprensión lectora, producción de textos y manejo de la lengua. Estas comparaciones se obtuvieron mediante la prueba t de Student y la prueba de Wilcoxon, la primera para las distribuciones normales y la segunda para aquellas que no presentaron una distribución normal.

Posteriormente, los puntajes brutos se ubicaron en los porcentajes de logros correspondientes, asignándoseles así un nivel de desempeño dentro de los cinco que establece la prueba.

\subsection{Trabajo de campo}

Antes de dar a conocer el trabajo de campo, es importante señalar que esta investigación surge como resultado de la adjudicación 
de un Proyecto de Investigación de la Universidad de Concepción. Dicho proyecto otorgó tiempo y recursos económicos a los integrantes del equipo investigador de la Universidad, quienes propusieron la innovación al colegio particular subvencionado.

Teniendo como marco orientador las Comunidades de Aprendizaje, esta investigación trabajó con grupos interactivos en aula, asociado a la incorporación de colaboradores de aprendizajes y considerando los principios del aprendizaje dialógico.

A continuación, se detallan las fases que se llevaron a cabo en esta intervención. Como ya se señaló, estas fases fueron modificadas y adaptadas al contexto del establecimiento donde se desarrolló la investigación:

a) Presentación (octubre, 2013): el equipo investigador de la Universidad presentó a la Comunidad Educativa del colegio los conceptos principales que orientan el trabajo con grupos interactivos y colaboradores de aprendizaje en aula.

b) Toma de decisión y conformación de equipos de trabajo (noviembre, 2013): una vez aprobada la incorporación de grupos interactivos por parte del colegio, se constituyó el equipo de trabajo entre la Universidad y el establecimiento educativo que participaría activamente en las fases posteriores. En este contexto, los pasos a seguir fueron consensuados por todos los agentes educativos involucrados.

c) Sensibilización del equipo involucrado (noviembre diciembre, 2013): se desarrollaron sesiones de reflexión con todo el equipo. En estas sesiones se abordaron temáticas relacionadas con los principios del aprendizaje dialógico y el cambio de roles que estos principios conllevan en relación al rol del docente y rol del alumno. Por otra parte, se abordó el cambio metodológico implicado en los grupos interactivos y la incorporación de colaboradores de aprendizaje en el aula.

d) Diálogos con los padres/madres (diciembre, 2013 - noviembre, 2014): esta fase se desarrolló incorporando a los padres y madres desde el inicio, a través de reuniones realizadas en el colegio, donde se les presentó el proyecto. En estas reuniones todos pudieron opinar y aclarar dudas, respecto a la manera en que ellos podrían formar parte de este trabajo. Finalmente, para el trabajo que siguió, fueron algunas de las madres quienes se comprometieron a participar, siendo agentes protagónicos dentro de estos grupos de trabajo. Además, junto con incorporarse semanalmente a los 
grupos interactivos, como colaboradoras, asistían a reuniones dialógicas semanales, en las que se planificaba el trabajo a realizar en los grupos interactivos.

e) Diagnóstico y contextualización (diciembre, 2013 - marzo, 2014): se realizó un diagnóstico de la realidad del colegio que se complementó con los antecedentes previos que se tenían del establecimiento. Estos datos se expresan en la descripción de la población y muestra, así como en los resultados del pretest CL-PT.

f) Planificación general (marzo, 2014): considerando los resultados del diagnóstico(CL-PTylos antecedentes entregados por los profesores y apoderados) y los requerimientos del MINEDUC, se planificó de manera general los aprendizajes que se abordarían durante el año. Se formaron grupos de trabajo, compuestos por los colaboradores de aprendizajes, quienes desarrollaron reuniones para determinar las acciones de los aspectos a transformar.

g) Conformación de grupos interactivos y reuniones dialógicas (abril-noviembre, 2014): estos grupos interactivos se desarrollaron para la asignatura de Lenguaje de manera progresiva (una vez por semana desde abril a julio y dos veces por semana desde agosto a noviembre). Para desarrollar esto, se formaban cuatro grupos interactivos de manera simultánea en el aula. Cada uno de estos grupos se encontraba tutorizado por un colaborador de aprendizaje (profesor, madre, estudiante de curso superior, alumna de pregrado o docente de la Universidad), quien les presentaba un material y/o actividad diferente a los grupos. Se utilizó, en general, material concreto y lúdico. La duración del trabajo en cada uno de estos grupos era de quince minutos aproximadamente. Una vez finalizado este tiempo, los estudiantes rotaban a un nuevo grupo y así hasta pasar por los cuatro grupos interactivos. Cada una de estas sesiones tuvo una duración de dos horas pedagógicas (lo que equivale a una hora y media cronológica). Las actividades propuestas en los cuatro grupos se determinaban con un objetivo de aprendizaje común (señalados por el MINEDUC para lenguaje en el nivel de primero básico), con el propósito de que los niños tuvieran la posibilidad de vivenciar una diversidad de oportunidades de aprendizaje en una misma clase, logrando afianzar estos. A su vez, se realizaron semanalmente reuniones dialógicas de evaluación 
y planificación con los colaboradores de aprendizaje, con el fin de ir mejorando la praxis. Estas reuniones permitían reflexionar en torno a lo realizado en los grupos interactivos, repensando las fortalezas y debilidades, considerando la opinión de todos los colaboradores de aprendizaje (con base en el diálogo igualitario) para dinamizar y mejorar de manera permanente la ejecución del trabajo.

\section{RESULTADOS Y SU ANÁLISIS}

La entrega de resultados comprende dos aspectos: en primer lugar, un análisis de puntajes brutos que implica la aplicación de pruebas de normalidad y estadísticos de pruebas. En segundo lugar, un análisis de porcentajes de logros y nivel de desempeño correspondiente.

\subsection{Análisis de puntajes brutos, pruebas de normalidad y estadísticos de prueba}

A continuación, se presentan los resultados obtenidos, por niño, expresados en puntajes brutos de la prueba CL-PT, pre- y postest.

GRÁFICO 2 - Puntajes totales por niño pre- y postest CP-LP (0-77puntos)

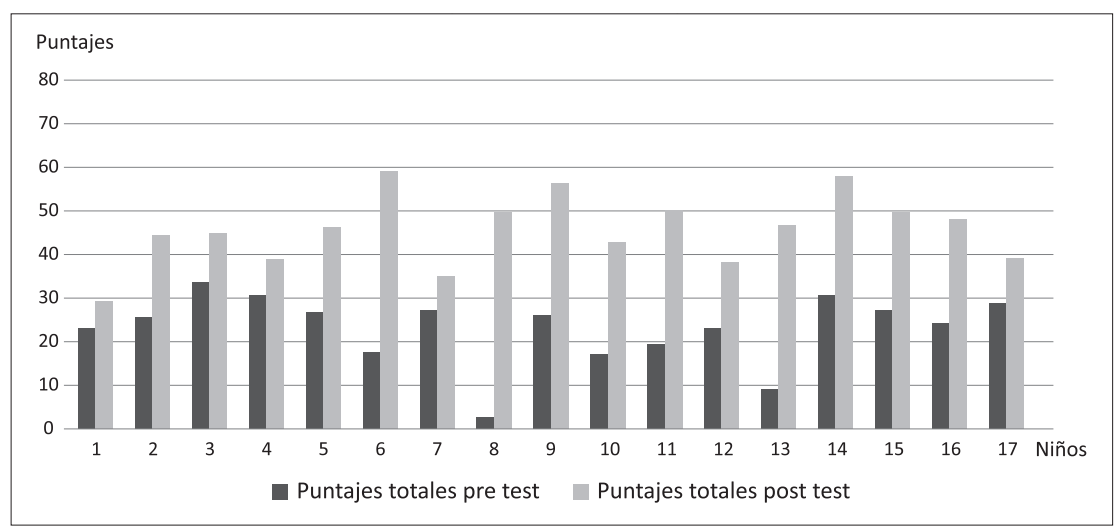

Fuente: Elaboración propia.

El Gráfico 2 muestra las diferencias de los puntajes brutos obtenidos por cada uno de los niños entre el pre- y postest. Se aprecian diferencias evidentes entre el pre- y postest en la mayoría de los estudiantes, quienes, en su totalidad, tuvieron un aumento desde la primera evaluación. Se observa, además, que, en el postest, la mayoría de los estudiantes se encuentra sobre la línea de los 40 puntos y solo uno de 
ellos se ubica bajo los 30 puntos en la segunda evaluación. Se destacan diferencias evidentes de los puntajes pre- y postest de los niños 6,8 y 13, quienes pasaron de estar bajo los 20 puntos a estar sobre los 40 puntos.

La Tabla 1 presenta los resultados de las pruebas de normalidad que se llevaron a cabo, para determinar el tipo de distribución de la muestra.

TABLA 1 - Pruebas de normalidad pre- y postest CL-PT

\begin{tabular}{|l|r|r|r|r|r|r|}
\hline \multirow{2}{*}{$\begin{array}{l}\text { Pruebas de } \\
\text { normalidad }\end{array}$} & \multicolumn{3}{|c|}{ Kolmogorov-Smirnov } & \multicolumn{3}{c|}{ Shapiro-Wilk } \\
\cline { 2 - 7 } & Estadístico & $\mathrm{gl}$ & Sig. & Estadístico & $\mathrm{gl}$ & Sig. \\
\hline $\begin{array}{l}\text { Puntaje total } \\
\text { pretest CL-PT }\end{array}$ &, 206 & 17 &, 054 &, 894 & 17 &, 054 \\
\hline $\begin{array}{l}\text { Puntaje total } \\
\text { postest CL-PT }\end{array}$ &, 107 & 17 &, $200^{*}$ &, 975 & 17 &, 898 \\
\hline
\end{tabular}

Leyenda:

a. Corrección de la significación de Lilliefors

". Este es un límite inferior de la significación verdadera.

Fuente: Elaboración propia.

Se observa que la distribución en el puntaje total del pretest CLPT sigue una distribución normal dado el valor $-\mathrm{p}=0,054$ donde valor $-\mathrm{p}>0.05$, al igual que el puntaje total del pretest cuyo valor $-\mathrm{p}=0,898$.

TABLA 2 - Estadísticos descriptivos

\begin{tabular}{|l|c|r|r|r|r|}
\hline & N & Mínimo & Máximo & Media & Desv. típ. \\
\hline Puntaje total pretest CL-PT & 17 & 3,0 & 33,5 & 23,000 & 7,8581 \\
\hline Puntaje total postest CL-PT & 17 & 29,0 & 58,5 & 45,412 & 8,0044 \\
\hline N válido (según lista) & 17 & & & & \\
\hline
\end{tabular}

Fuente: Elaboración propia.

Los datos de la Tabla 2 muestran la distribución de los puntajes del pretest con un rango entre 3,0 y 33,5 puntos donde se obtiene en promedio 23 puntos (D.T $=7,8581$ ); y el postest con un rango entre $29 \mathrm{y}$ 58,5 puntos, y con media de 45,412 puntos (D.T $=8,0044$ puntos). 
GRÁFICO 3 - Distribución de puntajes pre- y postest CL-PT (0-77 puntos)

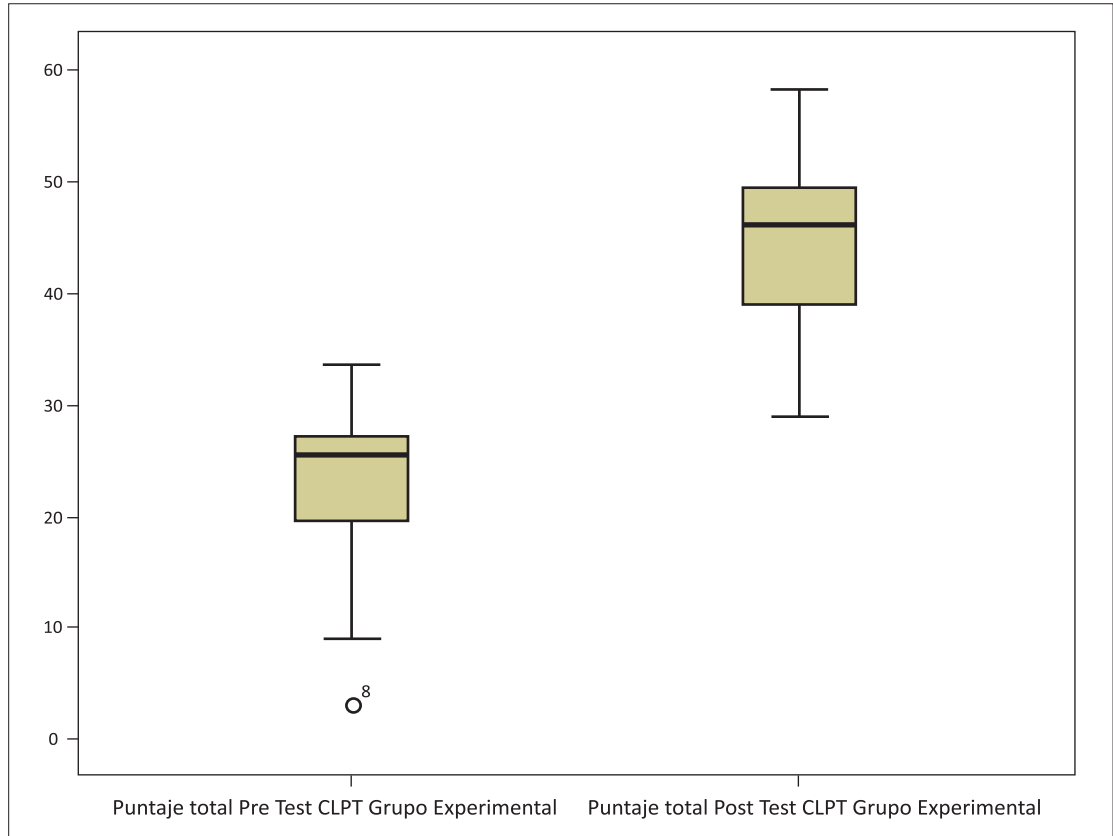

Fuente: Elaboración propia.

En el Gráfico 3 se observa que la distribución de los puntajes del pretest es distinta a la distribución de los puntajes del postest. La distribución de los puntajes del postest es mayor que la primera prueba aplicada.

Por lo tanto, para determinar si esta diferencia es significativa, se realiza prueba t de Student.

TABLA 3 - Prueba $\mathrm{t}$

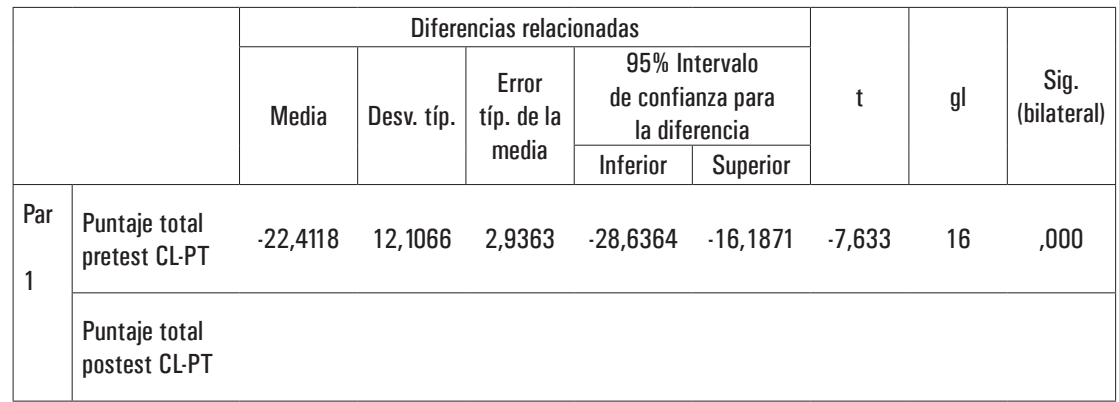

Fuente: Elaboración propia.

Según se observa en la Tabla 3, se puede señalar que se ha producido un aumento significativo $(\mathrm{t}=-7,633)$ en la evaluación de Comprensión Lectora. Esto viene a apoyar que el trabajo de los 
grupos interactivos permitió a los alumnos superar dificultades y obstáculos que se presentaban al inicio de la experiencia.

A continuación, se presenta un análisis detallado por dimensiones.

\section{DIMENSIÓN COMPRENSIÓN LECTORA}

La Tabla 4 presenta los resultados de las pruebas de normalidad que se llevaron a cabo para determinar el tipo de distribución de la muestra.

TABLA 4 - Pruebas de normalidad pre- y postest dimensión comprensión lectora

\begin{tabular}{|l|r|c|c|c|c|c|}
\hline & \multicolumn{3}{|c|}{ Kolmogorov-Smirnov } & \multicolumn{3}{c|}{ Shapiro-Wilk } \\
\cline { 2 - 8 } & Estadístico & $\mathrm{gl}$ & Sig. & Estadístico & $\mathrm{gl}$ & Sig. \\
\hline $\begin{array}{l}\text { Puntaje pretest } \\
\text { comprensión } \\
\text { lectora }\end{array}$ &, 141 & 17 &, $200^{*}$ &, 937 & 17 &, 287 \\
\hline $\begin{array}{l}\text { Puntaje postest } \\
\text { comprensión } \\
\text { lectora }\end{array}$ &, 130 & 17 &, $200^{*}$ &, 969 & 17 &, 801 \\
\hline
\end{tabular}

Leyenda:

a. Corrección de la significación de Lilliefors

". Este es un límite inferior de la significación verdadera.

Fuente: Elaboración propia.

Se observa que la distribución del puntaje del pretest para la dimensión comprensión lectora sigue una distribución normal dado el valor $-\mathrm{p}=0,287$ donde valor $-\mathrm{p}>0.05$, al igual que el puntaje del postest, cuyo valor $-\mathrm{p}=0,801$. Posteriormente, para determinar si esta diferencia es significativa, se realiza prueba t de Student.

TABLA 5 - Prueba t

\begin{tabular}{|c|c|c|c|c|c|c|c|c|c|}
\hline & & \multicolumn{5}{|c|}{ Diferencias relacionadas } & \multirow{3}{*}{$\mathrm{t}$} & \multirow{3}{*}{ gl } & \multirow{3}{*}{$\begin{array}{c}\text { Sig. } \\
\text { (bilateral) }\end{array}$} \\
\hline & & \multirow[t]{2}{*}{ Media } & \multirow[t]{2}{*}{$\begin{array}{c}\text { Desviación } \\
\text { típ. }\end{array}$} & \multirow{2}{*}{$\begin{array}{l}\text { Error } \\
\text { típ. de la } \\
\text { media }\end{array}$} & \multicolumn{2}{|c|}{$\begin{array}{c}95 \% \text { Intervalo } \\
\text { de confianza para } \\
\text { la diferencia }\end{array}$} & & & \\
\hline & & & & & Inferior & Superior & & & \\
\hline \multirow[t]{2}{*}{ Par } & $\begin{array}{l}\text { Puntaje } \\
\text { pretest } \\
\text { comprensión } \\
\text { lectora }\end{array}$ & $-9,8824$ & 5,9648 & 1,4467 & $-12,9492$ & $-6,8155$ & $-6,831$ & 16 & ,000 \\
\hline & $\begin{array}{l}\text { Puntaje } \\
\text { postest } \\
\text { comprensión } \\
\text { lectora }\end{array}$ & & & & & & & & \\
\hline
\end{tabular}

Fuente: Elaboración propia. 
Según se observa en la Tabla 5 , existe evidencia para señalar que se ha producido un aumento significativo (valor $-\mathrm{p}=0,000)$ en la dimensión comprensión lectora.

El puntaje promedio en el pretest en la dimensión comprensión lectora es de 14,882 puntos (D.T $=4,453$ ) y en el postest el puntaje promedio es de 24,765 puntos (D.T $=4,7405)$.

\section{DIMENSIÓN PRODUCCIÓN DE TEXTOS}

La Tabla 6 presenta los resultados de las pruebas de normalidad que se llevaron a cabo para determinar el tipo de distribución de la muestra.

TABLA 6 - Prueba de normalidad

\begin{tabular}{|l|r|r|r|r|r|r|}
\hline & \multicolumn{4}{|c|}{ Kolmogorov-Smirnova } & \multicolumn{3}{c|}{ Shapiro-Wilk } \\
\cline { 2 - 7 } & Estadístico & gl & Sig. & Estadístico & gl & Sig. \\
\hline $\begin{array}{l}\text { Puntaje pretest } \\
\text { producción de } \\
\text { textos }\end{array}$ & 173 & \multicolumn{10}{c}{17} &, 186 &, 890 & 17 &, 046 \\
\hline $\begin{array}{l}\text { Puntaje postest } \\
\text { producción de } \\
\text { textos }\end{array}$ &, 171 & 17 &, $200^{*}$ &, 964 & 17 &, 708 \\
\hline
\end{tabular}

Leyenda:

a. Corrección de la significación de Lilliefors

:Este es un límite inferior de la significación verdadera.

Fuente: Elaboración propia.

Se observa que la distribución del puntaje del pretest para la dimensión producción de textos no sigue una distribución normal dado el valor $-\mathrm{p}=0,046$ donde valor $-\mathrm{p}<0.05$. Luego se decide aplicar test de Wilcoxon para determinar si existen diferencias entre ambas pruebas.

TABLA 7 - Prueba de los rangos con signo de Wilcoxon

\begin{tabular}{|l|l|}
\hline \multicolumn{2}{|c|}{ Estadísticos de contraste $^{\mathrm{a}}$} \\
\hline & $\begin{array}{l}\text { Puntaje postest producción de textos en grupo experimental Puntaje pretest producción } \\
\text { de textos en grupo experimental }\end{array}$ \\
\hline Z & $, 1,392^{\mathrm{b}}$ \\
\hline $\begin{array}{l}\text { Sig. asintót. } \\
\text { (bilateral) }\end{array}$ &, 164 \\
\hline
\end{tabular}

Leyenda:

a. Prueba de los rangos con signo de Wilcoxon.

b. Basado en los rangos negativos.

Fuente: Elaboración propia. 
Según se observa en la Tabla 7 , se puede señalar que, en relación a la dimensión producción de textos, no se produjo un aumento significativo. Estos resultados pueden deberse a que esta dimensión se refiere a una actividad de mayor complejidad que requiere de un desarrollo cognitivo superior, así como de microhabilidades específicas, que, en el caso del grupo de alumnos de primero básico, no estaban desarrolladas, y que requerirían de un tiempo mayor de trabajo. En la dimensión producción de textos, se obtiene en promedio 7,882 puntos (D.T $=5,1068$ ) en cambio en el puntaje promedio del postest es de 11,206 puntos $(\mathrm{D} . \mathrm{T}=5,7989)$.

\section{DIMENSIÓN: MANEJO DE LA LENGUA}

La Tabla 8 presenta los resultados de las pruebas de normalidad que se llevaron a cabo para determinar el tipo de distribución de la muestra.

TABLA 8 - Pruebas de normalidad

\begin{tabular}{|l|r|r|r|r|r|r|}
\hline & \multicolumn{3}{|c|}{ Kolmogorov-Smirnov $^{\mathrm{a}}$} & \multicolumn{3}{c|}{ Shapiro-Wilk } \\
\cline { 2 - 7 } & Estadístico & $\mathrm{gl}$ & \multicolumn{1}{c|}{ Sig. } & Estadístico & $\mathrm{gl}$ & \multicolumn{1}{c|}{ Sig. } \\
\hline $\begin{array}{l}\text { Puntaje pretest } \\
\text { manejo de la } \\
\text { lengua en grupo } \\
\text { experimental }\end{array}$ &, 521 & 17 &, 000 &, 385 & 17 &, 000 \\
\hline $\begin{array}{l}\text { Puntaje postest } \\
\text { manejo de la } \\
\text { lengua en grupo } \\
\text { experimental }\end{array}$ &, 191 & 17 &, 100 &, 890 & 17 &, 046 \\
\hline
\end{tabular}

Leyenda:

a. Corrección de la significación de Lilliefors

Fuente: Elaboración propia.

Se observa que la distribución del puntaje del pretest para la dimensión manejo de la lengua no sigue una distribución normal dado el valor $-\mathrm{p}=0,000$ donde valor $-\mathrm{p}<0.05$. Luego se decide aplicar test de Wilcoxon para determinar si existen diferencias entre ambas pruebas. 
TABLA 9 - Prueba de los rangos con signo de Wilcoxon

\begin{tabular}{|l|l|}
\hline \multicolumn{2}{|c|}{ Estadísticos de contraste $^{\mathrm{a}}$} \\
\hline & $\begin{array}{l}\text { Puntaje postest manejo de la lengua en grupo experimental } \\
\text { Puntaje pretest manejo de la lengua en grupo experimental }\end{array}$ \\
\hline Z & $-3,630^{\mathrm{b}}$ \\
\hline $\begin{array}{l}\text { Sig. asintót. } \\
\text { (bilaterall) }\end{array}$ &, 000 \\
\hline
\end{tabular}

Leyenda:

a. Prueba de los rangos con signo de Wilcoxon

b. Basado en los rangos negativos

Fuente: Elaboración propia.

En la Tabla 9 se evidencia que existe un aumento significativo (valor $-p=0,00$ ) en el puntaje obtenido por los alumnos en esta dimensión. En la dimensión manejo de la lengua, se obtiene en promedio 0,235 puntos (D.T $=0,6642$ ) en cambio en el puntaje promedio del postest es de 9,441 puntos (D.T $=2,37$ ).

En síntesis, se puede señalar que la prueba CL-PT mostró un avance significativo en los puntajes globales. Lo mismo ocurrió con las dimensiones comprensión lectora y manejo de la lengua. En el caso de la dimensión producción de textos, si bien hubo un avance, este no fue significativo.

\subsection{Análisis de porcentajes de logros y nivel de desempeño}

Luego de realizar un análisis de los puntajes brutos, se transformaron estos a porcentaje de logro y su correspondiente nivel de desempeño, según la tabla propuesta (ver Cuadro 1 de MEDINA; GAJARDO, 2012).

GRÁFICO 4 - Porcentajes de logro e nivel de desempeño CL-PT por niño pre- y postest

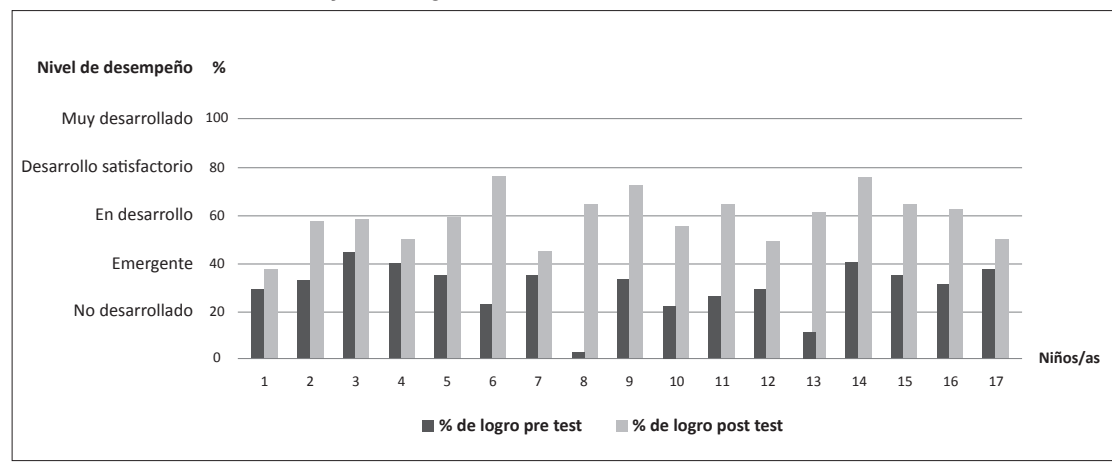

Fuente: Elaboración propia. 
En el Gráfico 4 se muestran los porcentajes obtenidos por los estudiantes evaluados. Se evidencia un desplazamiento desde el nivel "Emergente", en el que se encontraban en el pretest, hacia los dos niveles siguientes, a excepción de uno (niño 1), que permanece en "Emergente" con un desplazamiento positivo de su porcentaje de logro dentro del nivel. Se puede observar, además, un desplazamiento positivo importante de dos estudiantes (8 y 13), que avanzan desde el nivel "No Desarrollado" hasta "Desarrollo Satisfactorio" luego de la incorporación de grupos interactivos.

GRÁFICO 5 - Porcentajes de logro y nivel de desempeño por dimensión

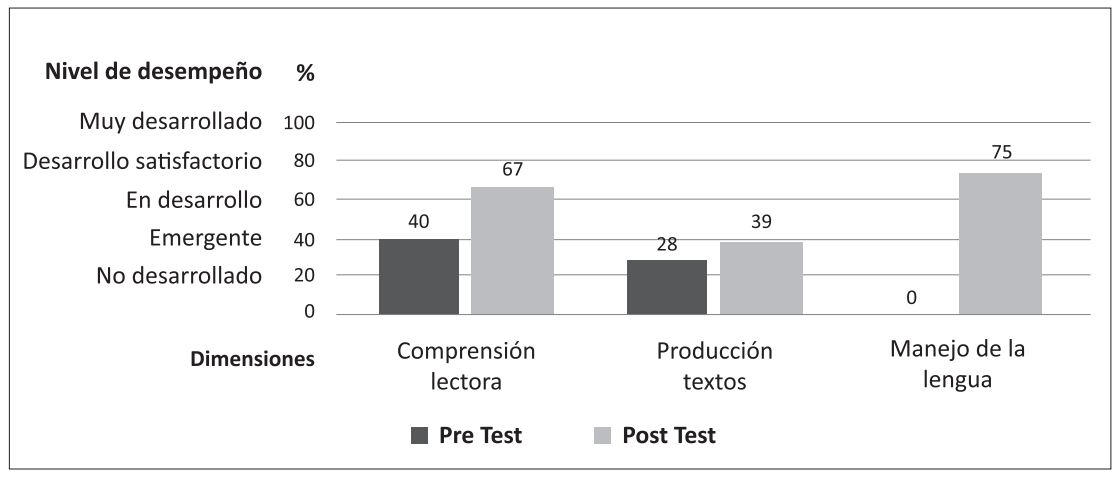

Fuente: Elaboración propia.

El Gráfico 5 muestra las diferencias del pre- y postest entre los porcentajes de logro y el nivel de desempeño según las dimensiones evaluadas (comprensión lectora, producción de textos y manejo de la lengua). Se aprecia, nuevamente, un incremento evidente en cada una de las dimensiones, donde se destaca la diferencia entre el pre- y postest de la dimensión manejo de la lengua, que pasa de estar en el nivel "No Desarrollado" al nivel de "Desarrollo Satisfactorio", con el porcentaje más alto de las tres dimensiones. Le sigue comprensión lectora, que también se ubica en un "Desarrollo Satisfactorio", avanzando un nivel desde el pretest. La dimensión producción de textos, como ya se evidenció en la prueba de significancia, no muestra un avance significativo en relación al pretest y al postest, si bien se observa un avance, no superan el nivel "Emergente". En este sentido, como ya se apuntó, hay que considerar que la habilidad de escritura, por la complejidad de factores implicados en su desarrollo, requiere de un tiempo más extenso para su apropiación cabal (MEDINA; GAJARDO, 2012). 


\section{CONCLUSIONES}

Con respecto al objetivo determinar el posible incremento en comprensión lectora en niños de primero básico, luego de la incorporación de grupos interactivos en el aula, podemos señalar que, por una parte, se produjo un incremento significativo entre los puntajes en esta dimensión entre el pre- y postest, que se evidencia en la prueba de significancia, que arrojó un valor $-\mathrm{p}=0,000$. Por otra parte, los porcentajes de logro en esta dimensión variaron entre un $40 \%$ del pretest a un $67 \%$ en el post test, obteniéndose un incremento desde un nivel "Emergente" a un nivel de "Desarrollo Satisfactorio".

En relación al objetivo determinar el posible incremento en producción de textos, podemos señalar que se produjo un aumento de puntaje, pero este no fue significativo, con un valor $-\mathrm{p}=0,164$. Esto puede deberse, entre otros factores, al hecho de que las habilidades necesarias para logro de la competencia de escritura requieren de ejercitación por un periodo más largo. Lo que refleja que, si bien se avanzó en el desarrollo de producción de textos en los niños, falta afianzar ciertas habilidades de escritura. Es así como los niños se mantuvieron en el nivel "Emergente", sin embargo, hubo un avance, ya que se desplazaron entre un $28 \%$ en el pretest a un $39 \%$ en el postest.

En torno al objetivo determinar el posible incremento en manejo de la lengua, podemos plantear que hubo un incremento significativo de puntajes entre el pre- $\mathrm{y}$ postest, con un valor $-\mathrm{p}=0,00$. Los porcentajes de logro en esta dimensión avanzaron entre un $0 \%$ en pretest a un $75 \%$ en el postest, obteniéndose un incremento desde un nivel de desempeño "No Desarrollado" a un nivel de "Desarrollo Satisfactorio".

Finalmente, podemos establecer que los resultados obtenidos en las pruebas aplicadas demuestran un incremento en el desarrollo de las habilidades lingüísticas del grupo de estudiantes en el cual se realizó la intervención con grupos interactivos. En este sentido y como ya se señaló, los resultados muestran a un grupo importante de niños que avanza desde los niveles "Emergente" y "No Desarrollado" a un nivel de desempeño con "Desarrollo Satisfactorio", en las tres dimensiones, lo que indica un "predominio de fortaleza sobre las necesidades de aprendizaje." (MEDINA; GAJARDO, 2012, p. 82). Es importante destacar que, en el caso del grupo de niños estudiado, el acceso temprano a la cultura escrita es casi inexistente y es únicamente mediado por el colegio. Esto genera una gran brecha de la que se hace cargo el establecimiento. En este contexto, el trabajo con grupos interactivos, al tomar en cuenta las falencias individuales de los estudiantes y ofrecer diversidad de oportunidades 
de aprendizaje, logra incrementar los niveles de desarrollo que los niños tienen, llevándolos a superar las diversas limitaciones impuestas por sus contextos familiares. Además, el grupo de niños que logra alcanzar "Desarrollo Satisfactorio" demuestra que las capacidades de los estudiantes son óptimas, pero requieren de permanentes apoyos para desplegar sus potencialidades.

\section{AGRADECIMIENTO}

El equipo de Investigación agradece a la Vicerrectoría de Investigación y Desarrollo (VRID) de la Universidad de Concepción por el financiamiento del Proyecto VRID código 213.421.001-1.0.

\section{REFERENCIAS}

AGENCIA DE CALIDAD DE LA EDUCACIÓN. Informe de Resultados Simce 2. Básico 2013 para Docentes y Directivos. Santiago: Gobierno de Chile, 2014. Disponible en: http://www.agenciaeducacion.cl/evaluaciones/que-es-el-simce/. Consultado el: 23 jul. 2014.

AGUILAR, C. et al. Lectura dialógica y transformación en las comunidades de aprendizaje. Revista Interuniversitaria de Formación de Profesorado, Zaragoza, v. 24, n. 67, p. 31-44, abr. 2010.

ALLIENDE, F.; CONDEMARÍN, M. La lectura: teoría, evaluación y desarrollo. Santiago: Andrés Bello, 2002.

AUBERT, A. et al. Aprendizaje dialógico en la sociedad de la información. Barcelona: Hipatía, 2010.

BAROCCIO, R. La resistencia al cambio: Estrategias no convencionales para construir apoyo para el cambio. México: Facultad de Psicología UNAM, 2000, citado por GUERRERO, P. Estudio de las resistencias de los profesores a una estrategia para el desarrollo de la creatividad en tres unidades educativas. Psykhe, Santiago, v. 14, n. 1, p. 31-45, mayo. 2005. Disponible en: < http://dx.doi.org/10.4067/S0718-22282005000100003>. Consultado el: 07sept. 2015. BURBULES, N. El diálogo en la enseñanza: teoría y práctica. Buenos Aires: Amorrortu, 1999. CASSANY, D.; LUNA, M.; SANZ, G. Enseñar lengua. Barcelona: Graó, 2008.

CLARK, C.; AKERMAN, R. Social inclusion and reading: an exploration. National Literacy Trust, June, 2006. Disponible en: < http://www.literacytrust.org.uk/assets/0000/0566/ Social_inclusion_reading_2006.pdf>. Consultado el: 05 Oct. 2015.

DÍEZ, C. La escritura colaborativa en educación infantil: estrategias para el trabajo en el aula. Barcelona: Horsori, 2004.

ECHEVERRÍA, E. Filosofía para niños. México: SM, 2004.

EISTERHOLD, J. "Reading-writing connections: Toward a description for second language learners". En B. Kroll (ed.) Second language writing: Research insights for the classroom (p.88-101). Cambridge: Cambrigne University Press, 1991, citado por PARODI, G. Comprensión de textos escritos. Buenos Aires: Eudeba, 2005.

ELBOJ, C. et al. Comunidades de Aprendizaje: transformar la educación. Barcelona: Graó, 2009. FERRADA, D. Construyendo escuela, compartiendo esperanzas: la experiencia del proyecto "Enlazando Mundos". Santiago: Ril, 2012. 
FERRADA, D. "Enlazando mundos": un modelo pedagógico que construye esperanzas de igualdad e inclusión en escuelas públicas. Rexe, Concepción, v. 7, n. 14, p. 37-52, agosto-dic. 2008. FERRADA, D.; FLECHA, R. El modelo dialógico de la pedagogía: un aporte desde las experiencias de comunidades de aprendizaje. Estudios Pedagógicos, Valdivia, v. 34, n. 1, p. 41-61, mes. 2008. Disponible en: https://dx.doi.org/10.4067/S0718-07052008000100003 Consultado el: 10 nov. 2014.

FLECHA, R. Compartiendo palabras: el aprendizaje de las personas adultas a través del diálogo. Barcelona: Paidós, 1997.

FLECHA, R.; LARENA, R. Comunidades de aprendizaje. Sevilla: Fundación ECOEM, 2008.

FONS, M. Leer y escribir para vivir: alfabetización inicial y uso real de la lengua escrita en la escuela. Barcelona: Graó, 2004.

FREIRE, P. Pedagogía del oprimido. Madrid: Siglo XXI, 1970.

GUERRERO, P. Estudio de las resistencias de los profesores a una estrategia para el desarrollo de la creatividad en tres unidades educativas. Psykhe, Santiago, v. 14, n. 1, p. 31 45, mayo. 2005. Disponible en: < http://dx.doi.org/10.4067/S0718-22282005000100003>. Consultado el: 07 sept. 2015.

INOSTROZA, G. Aprender a formar niños lectores y escritores. Santiago: Dolmen,1996. JOLIBERT, J. Formar niños lectores de textos. Santiago: LOM, 2003.

JOLIBERT, J.; SRAÏKI, C. Niños que construyen su poder de leer y escribir. Santiago: Manantial, 2009.

MEDINA, A.; GAJARDO, A. Pruebas de comprensión lectora y producción de textos. Santiago: Ediciones UC, 2012.

OLIVER, E.; GATT, S. De los actos comunicativos de poder a los actos comunicativos dialógicos en las aulas organizadas en grupos interactivos. Revista Signos, Valparaíso, v. 43, n. 2, p. 279-294, 2010. Disponible en: <http://www.scielo.cl/pdf/signos/v43s2/a02.pdf>. Consultado el: 13 oct. 2015.

ORGANIZACIÓN PARA LA COOPERACIÓN Y EL DESARROLLO ECONÓMICO - OCDE. Reading performance (PISA), 2012. Disponible en: < https://data.oecd.org/ pisa/reading-performance-pisa.htm>. Consultado el: 10 nov. 2014.

PARODI, G. Comprensión de textos escritos. Buenos Aires: Eudeba, 2005.

SHANAHAN, T. "Nature of the reading-writing relation: an exploratory multivariate analysis". Journal of Educational Psychology, Washington, DC. v. 2, n. 78,1984. Citado por PARODI, G. Comprensión de textos escritos. Buenos Aires: Eudeba, 2005.

TOLCHINSKY,L. Aprendizaje del lenguaje escrito: procesos evolutivos e implicaciones didácticas. Barcelona: Anthropos, 1993. Citado por DÍEZ, C. La escritura colaborativa en educación infantil: estrategias para el trabajo en el aula. Barcelona: Horsori, 2004.

VAN DIJK, T.; KINTSCH, W. "Toward a model of text comprehension and production". Psychological Review, Washington, DC n.85 p.363-394, 1978. Citado por PARODI, G. Comprensión de textos escritos. Buenos Aires: Eudeba, 2005

VALLS, R. Comunidades de aprendizaje. Una práctica educativa de aprendizaje dialógico para la sociedad de la información. Tesis doctoral no publicada. Barcelona: Universidad de Barcelona, 2000. Disponible en: http://www.tesisenred.net/handle/10803/2929. Consultado el: 16 abr. 2014.

VALLS, R.; KYRIAKIDES, L. The power of interactive groups: how diversity of adults 
volunteering in classroom groups can promote inclusion and success for children of vulnerable minority ethnic populations. Cambridge Journal of Education, Cambridge, v. 43, n. 1, p.17-33, Mar. 2013.

VELASCO, A.; ALONSO, L. Sobre la teoría de la educación dialógica. Educere, Mérida, n. 42, p. 461 - 470, jul. /sep. 2008.

\section{NOTAS}

${ }^{1}$ El artículo corresponde a los resultados derivados de un estudio realizado por la investigadora principal en el contexto de la Tesis doctoral del Programa de Doctorado en Educación de la Universidad de Sevilla, España.

${ }^{2}$ Vulnerable: en Chile se utiliza esta denominación para aludir a grupos sociales de riesgo. En el caso de los colegios "vulnerables", se asigna un valor que se calcula a través del IVE (Índice de Vulnerablidad del Establecimiento). Es un indicador del nivel de vulnerabilidad presente en cada establecimiento educativo. Se expresa en porcentaje, de 0 a $100 \%$, tomando en cuenta tres variables: años de escolaridad de la madre, años de escolaridad del padre e ingresos del hogar del alumno.

${ }^{3}$ Primer año básico: nivel educativo formal que involucra a los niños de 6 a 7 años en Chile.

${ }^{4}$ CL-PT: Prueba de Comprensión Lectora y Producción de Textos.

${ }^{5}$ Em situação de vulnerabilidade: no Chile, utiliza-se essa denominação para aludir a grupos sociais em situação de risco. No caso dos colégios em situação de vulnerabilidade, atribui-se a eles uma nota que se calcula através do IVE (Índice de Vulnerabilidade do Estabelecimento). É um indicador de nível de vulnerabilidade presente em cada estabelecimento educativo. Expressa-se em porcentagem, de 0 a 100\%, tendo por base três variáveis: anos de escolaridade da mãe, anos de escolaridade do pai e renda familiar do aluno.

${ }^{6}$ Primeiro ano básico: nível educativo formal que envolve crianças de 6 a 7 anos no Chile. Equivalente ao primeiro ano do Ensino Fundamental brasileiro (N.T.).

${ }^{7}$ CL-PT: Prova de Compreensão de Leitura e Produção de Textos.

${ }^{8}$ SIMCE: Sistema de Medición de la Calidad de la Educación en Chile.

${ }^{9}$ OCDE: Organización para la Cooperación y el Desarrollo Económico, a la cual Chile pertenece.

${ }^{10}$ Colegios particulares subvencionados son aquellos que tienen dependencia de privados y además apoyo económico fiscal del Ministerio de Educación de Chile.

${ }^{11}$ Colaboradores de aprendizaje: en adelante, para fines de este estudio, cuando se mencionen los colaboradores de aprendizaje, nos estaremos refiriendo a un grupo constituido por: madres, niños de cursos superiores del mismo colegio, alumnas de pregrado de las carreras de pedagogía y docentes de la Universidad, que colaboraron en este proyecto.

${ }^{12}$ Educación General Básica (EGB): Tiene una duración de ocho años y es el segundo nivel formal dentro del sistema educativo chileno después de la Educación Parvularia.

${ }^{13}$ NEE: Necesidades Educativas Especiales.

Presentado: 22/01/2016

Aprobado: $12 / 01 / 2017$ 
Dirección postal:

Juan Antonio Coloma 0201

Los Ángeles | Chile.

Código postal: 4451032 